\title{
Case Report \\ Overlooking the Obvious during the COVID-19 Pandemic: Dyspnoea with Asymmetric Breath Sounds in a Toddler
}

\author{
Heli Salmi iD, ${ }^{1,2}$ Heini Harve-Rytsälä, ${ }^{3}$ Paula Rautiainen, ${ }^{2}$ Sari Pyörälä, ${ }^{4}$ \\ and Johanna Hästbacka ${ }^{2}$ \\ ${ }^{1}$ Paediatric Research Center, New Children's Hospital, University of Helsinki and Helsinki University Hospital, Helsinki, Finland \\ ${ }^{2}$ Department of Anaesthesia and Intensive Care, New Children's Hospital, \\ University of Helsinki and Helsinki University Hospital, P.O. Box 347, Fi-00029 HUS, Finland \\ ${ }^{3}$ Emergency Medicine and Services, University of Helsinki and Helsinki University Hospital, P.O. Box 340, \\ FI-00029 HUS, Finland \\ ${ }^{4}$ Department of Paediatric Surgery, New Children's Hospital, University of Helsinki and Helsinki University Hospital, \\ P.O. Box 347, Fi-00029 HUS, Finland
}

Correspondence should be addressed to Heli Salmi; heli.salmi@hus.fi

Received 17 August 2020; Accepted 22 January 2021; Published 2 February 2021

Academic Editor: Miguel O Ryan

Copyright (c) 2021 Heli Salmi et al. This is an open access article distributed under the Creative Commons Attribution License, which permits unrestricted use, distribution, and reproduction in any medium, provided the original work is properly cited.

Background. Paediatric healthcare specialists are concerned about the secondary effects of the COVID-19 pandemic on children. We report a case of acute respiratory distress in a healthy toddler whose healthcare providers were sidetracked from the correct diagnosis by suspicion of COVID-19. Case Presentation. The patient was a 20-month-old healthy boy. In the morning, he had coughed while drinking milk. He was asymptomatic for the day but presented with acute respiratory distress when lying down in the evening. An ambulance was called, and he was taken to a tertiary hospital's paediatric emergency department, where his condition and oxygen saturation fluctuated. He had mildly elevated temperature and petechiae on his trunk, showed asymmetrical radiographic and auscultatory pulmonary findings, and did not tolerate any exertion. Pneumonia was suspected, SARS-CoV-2 was considered as potential causative agent, and the child was admitted to a Paediatric Intensive Care Unit. As the patient did not show clear signs of infection or bronchial obstruction, the events were thoroughly rediscussed with the caregiver next morning. It was then found out that the child had also been eating cashew nuts. Multiple pieces of cashew nuts were removed from the left bronchial tree in a bronchoscopy. After the procedure, all symptoms promptly resolved. Foreign body aspiration-an obvious cause of acute respiratory distress in our patient's age group-was overlooked by experienced emergency medical care providers and paediatric critical care physicians due to the slightly unusual presentation, incomplete anamnestic information, and a bias to consider COVID-19 in the current exceptional circumstances. Conclusions. Emergency care providers are instructed to consider all patients with respiratory distress as potential COVID-19 patients. However, the clinical course of COVID-19 infection is usually mild in children. Therefore, alternative causes for serious breathing difficulty are more likely, and all differential diagnoses should be considered in the usual unbiased manner.

\section{Introduction}

Children seem to be spared from the most serious forms of coronavirus disease 2019 (COVID-19) [1-4] caused by the novel pandemic coronavirus SARS-CoV-2. However, cases with significant symptoms and need for hospitalization including intensive care have been reported $[2,3]$. Coinfection with other airway pathogens, such as Mycoplasma pneumoniae and respiratory syncytial virus, has been reported in $6.5 \%$ [5] or as much as $51 \%$ [6] of children with COVID-19. Furthermore, since other infectious and noninfectious causes for dyspnoea and respiratory failure are more common in children than SARS-CoV-2, it is crucial to carefully evaluate all differential diagnoses in children with respiratory symptoms. The continuous presence of COVID19 in media may create an illusion of high prevalence of 
COVID-19 among children. Thus, diagnosis and treatment of common conditions may be delayed if caregivers avoid medical service contacts for fear of contracting COVID-19 and if healthcare professionals prioritize COVID-19 at the cost of delaying the diagnosis and treatment of more common conditions.

We report a case of rapidly deteriorating hypoxemic respiratory compromise in a 20-month-old toddler during the COVID-19 pandemic. We recognize that his prehospital and in-hospital treatments were probably influenced by the pandemic causing diagnostic delay.

\section{Case Presentation}

The patient was a previously healthy 20 -month-old boy. $\mathrm{He}$ had mild atopic eczema, with no history of asthma or wheezing in respiratory infections. He had been vaccinated according to the Finnish vaccination schedule, which includes vaccinations against Haemophilus influenzae, Bordetella pertussis, Streptococcus pneumoniae, and influenza. He had started day care the previous week. His parents and elder sibling were well and asymptomatic.

On the morning of the hospitalization, the boy had suddenly started to cough vigorously while drinking milk at breakfast. The coughing had ceased, and the boy had had an uneventful day at day care, playing, eating, and napping normally. In the evening, he had been irritable and reluctant to eat. He had gone to bed as usual but soon woken up with cough and apparent dyspnoea. His father had called an ambulance when noticing that he had turned blue in the face.

At arrival of the ambulance, the child was dyspnoeic, the oxygen saturation was $90 \%$, and the axial temperature was 38.5 C. The boy met the emergency medical services (EMS) criteria for suspicion of COVID-19, and he was transported to the emergency room (ER) with a notion "suspicion of COVID-19" and with the EMS personnel wearing personal protective equipment (PPE) according to the protocol.

Upon arrival at the ER, nasal high flow (NHF) oxygen was started and the patient was provided salbutamol by inhalation. A nasopharyngeal swab was taken for a SARSCoV-2 RNA test. The patient's oxygen saturation had risen to over $95 \%$ but dropped to $56 \%$ while establishing an intravenous line, as he became agitated. A paediatric critical care specialist was called in, and preparations for emergency intubation were started. The team dressed in full PPE. With $80-100 \%$ oxygen to NHF with $251 / \mathrm{min}$ flow $(21 / \mathrm{kg})$, the child was still breathing heavily with prolonged expiration but had an oxygen saturation of $93 \%$, with occasional desaturations to $60-70 \%$ while agitated. Breath sounds were absent from the left lower lung field. There were no rales or wheezing. The child was tachycardic (124-157/min) and febrile $(38.3 \mathrm{C})$, his capillary refill time was just in the normal range $(3 \mathrm{~s})$, peripheral pulses were well palpable, and blood pressure was normal $(110 / 79 \mathrm{mmHg})$. The child was conscious but lethargic, and petechiae were noted on his chest. A chest X-ray showed a diffuse opacity in the left lower lobe (Figure 1). The heart was not enlarged, and there was no pulmonary congestion. The laboratory findings, showing an

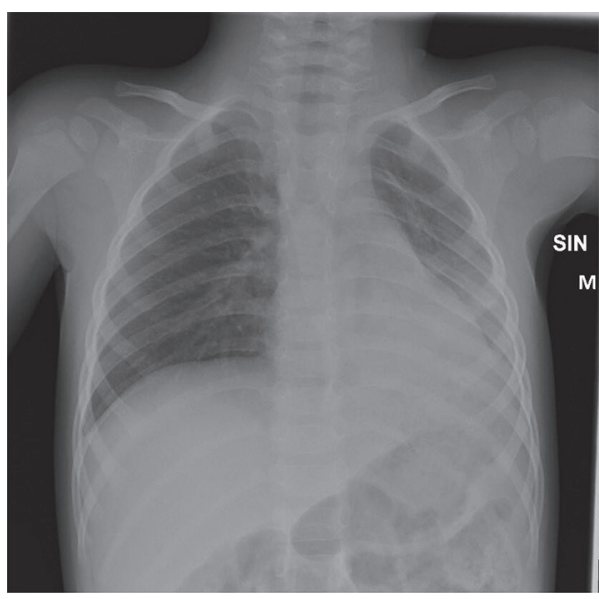

FIgURE 1: Chest X-ray at arrival, showing infiltrates and/or atelectasis in the left lower lobe.

elevated white cell count and hypoxaemia, are summarized in Table 1.

An intravenous fluid bolus was given, blood cultures were obtained, and intravenous ceftriaxone was administered. The child was transferred to a negatively pressurized isolation room in the Paediatric Intensive Care Unit (PICU). His breathing remained adequate on NHF with $70 \% \mathrm{FiO}_{2}$ and dexmedetomidine sedation. Arterial blood gases and lactate were in the normal range (Table 1). A bedside ultrasound examination showed a consolidated lung on the left side with no pleural effusion and normal heart contractility. During the ensuing 8 hours, there were no new organ dysfunctions, suggestive of sepsis, nor coughing or mucus secretions suggestive of pneumonia. However, in a follow-up chest X-ray, the whole left lung was atelectatic and the heart had shifted left (Figure 2). Breath sounds were absent on the left side.

The following morning, a reassessment of the rapidly evolved hypoxemic respiratory insufficiency with single lung involvement and without symptoms of an infection apart from mild fever led to a second interview with the mother. This revealed that in addition to drinking milk, the patient had also been eating cashew nuts.

A bronchoscopy was scheduled, and two large and several smaller pieces of cashew nuts were removed from the left main bronchus and the left upper lobe bronchus (Figures 3 and 4) two hours later. The atelectasis vanished, supplementary oxygen and respiratory support were promptly weaned, and the child was extubated on the same day. The SARS-CoV2 test was negative. There were no new petechiae; the ones noticed the previous evening were probably due to forceful coughing, or perhaps due to scratching his atopic eczema, as the mother suggested. The boy made a full and brisk recovery.

\section{Discussion and Conclusions}

Although COVID-19 appears to be generally mild or asymptomatic in children [1-3], the medical care children receive is negatively influenced by COVID-19 control 
TABLE 1: Laboratory values at arrival at night and the next morning.

\begin{tabular}{|c|c|c|c|}
\hline Laboratory test & Source & Result at arrival & Result $6 \mathrm{~h}$ after arrival \\
\hline Haemoglobin $(\mathrm{g} / \mathrm{L})$ & Blood & 131 & 121 \\
\hline White cell count $\left(10^{9} / \mathrm{L}\right)$ & Blood & $21.5(\mathrm{H})$ & 13.9 \\
\hline Neutrophil count $\left(10^{9} / \mathrm{L}\right)$ & Blood & 16.10 & 10.56 \\
\hline Platelet count $\left(10^{9} / \mathrm{L}\right)$ & Blood & 344 & 266 \\
\hline C-reactive protein $(\mathrm{mg} / \mathrm{dL})$ & Plasma & $<5$ & $13(\mathrm{H})$ \\
\hline Glucose $(\mathrm{mmol} / \mathrm{L})$ & Blood & 8.6 & 6.6 \\
\hline Lactate $(\mathrm{mmol} / \mathrm{L})$ & Arterial blood & 0.7 & 0.6 \\
\hline $\mathrm{pH}$ & Arterial blood & 7.40 & 7.39 \\
\hline Bicarbonate, standardized $(\mathrm{mmol} / \mathrm{L})$ & Arterial blood & 23 & 23 \\
\hline Base excess $(\mathrm{mmol} / \mathrm{L})$ & Arterial blood & -2.3 & -1.5 \\
\hline Oxygen, partial pressure $(\mathrm{kPa})$ & Arterial blood & $10.0(\mathrm{~L})$ & $15.9(\mathrm{H})$ \\
\hline Carbon dioxide, partial pressure $(\mathrm{kPa})$ & Arterial blood & 4.8 & 5.1 \\
\hline Prothrombine time (\% of normal) & Arterial blood & - & 74 \\
\hline
\end{tabular}

$\mathrm{H}$ (high) indicates a value higher than normal and $\mathrm{L}$ (low) indicates lower.

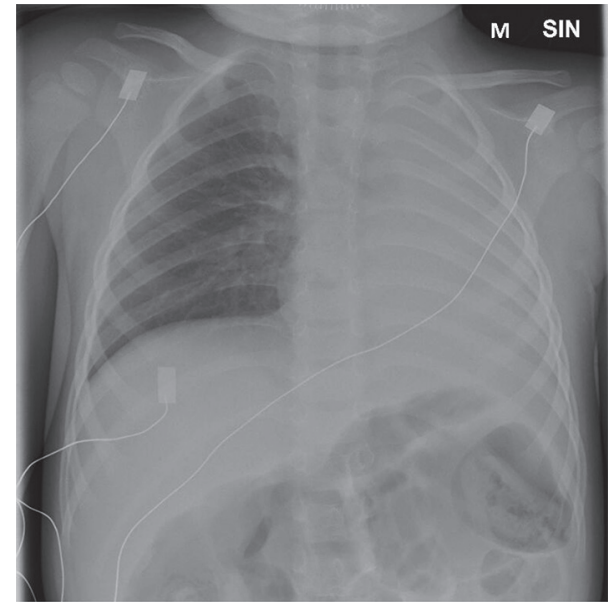

FIGURE 2: Follow-up chest X-ray $12 \mathrm{~h}$ after arrival. The left lung is completely atelectatic, and the mediastinum has shifted to the left.

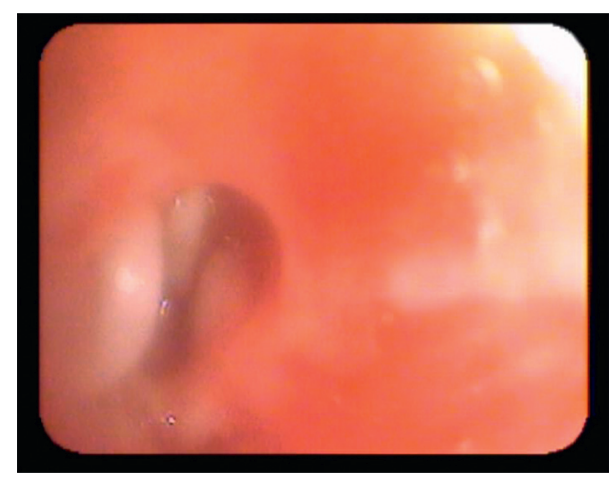

FIGURE 3: A still frame from the bronchoscopy, showing the foreign material in the left main bronchus.

measures [7-9]. As patient flows and customary protocols are abruptly modified and massive media coverage results in a generalized attention shift, healthcare professionals may make diagnostic deviations by considering COVID-19 over other more probable diagnoses. Furthermore, parents may not dare to bring even severely ill children to the hospital for fear of contracting COVID-19. Consequently, paediatric

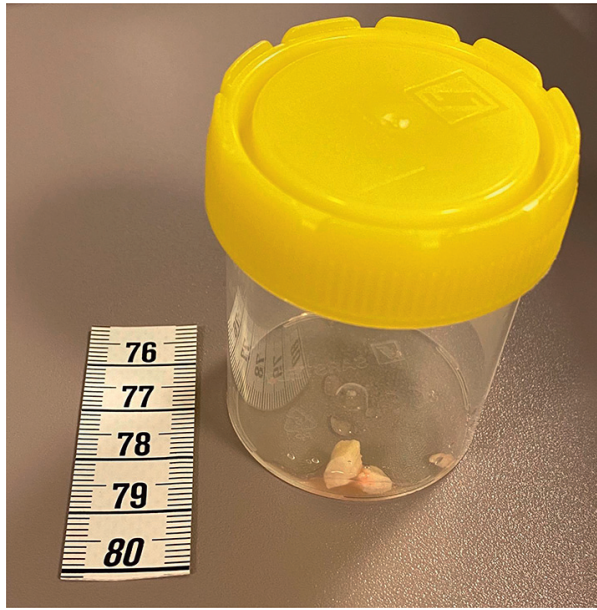

Figure 4: Two pieces of cashew nuts extracted from the left main bronchus.

healthcare providers have repeatedly expressed their concern about children becoming "secondary victims" of the COVID-19 pandemic [7-9].

Bronchial foreign body aspiration is a common cause of acute respiratory distress in children, particularly those aged three years or under. The left bronchus is affected less often than the right one. Diagnosis is often delayed due to the frequent symptomless period following the aspiration. The obstruction of the airway may worsen due to gradual swelling of the mucosa or swelling of the obstructing foreign object [10-12].

During a local epidemic peak of COVID-19 in the Helsinki University Hospital region, the prevalence of COVID-19 in children requiring EMS or ER care for infectious symptoms has been very low. Between 20/4/2020 and 20/5/2020, EMS contacted 358 children (0-15 years), of which $31(3.7 \%)$ presented with symptoms leading to a SARS-CoV-2 RNA test. All 31 tests turned out negative. In a separate analysis for prevalence of SARS-CoV-2 in children requiring secondary or tertiary ER, 3/113 (2.7\%) children (0-15 years) contacting a HUS ER with infectious symptoms between 8/4/2020 and 15/4/2020 were positive for SARS- 
CoV-2 RNA. These cohorts are small but regionally inclusive as HUS is the only provider of EMS as well as secondary- and tertiary-level ER care for children in the area. Consequently, even during a local epidemic peak of COVID-19, diagnostic measures should not overlook other common aetiologies for acute respiratory distress in order to avoid unnecessary delays in treatment.

In this case, EMS, ER, and PICU personnel-all dressed up in full PPE against COVID-19-consecutively overlooked a probable diagnosis in any toddler with acute onset dyspnoea, unilateral absence of breathing sounds, and unilateral lung atelectasis. The COVID-19 preparedness including PPE and alterations in usual protocols certainly caused an attention shift in the experienced professionals even when COVID-19 was not necessarily suspected as the primary diagnosis. The diagnosis may also have been delayed as the aspiration had not occurred to the conventional right side and as the patient displayed petechiae.

To conclude, it is valuable to note that foreign objects are not always aspirated to the right bronchus; petechiae can exist without sepsis; and in case of a critically ill child, any exceptional circumstances, such as those during the COVID-19 pandemic, should not distract from paying attention to the typical and critical aetiologies.

\section{Abbreviations}

COVID-19: Coronavirus disease 2019

ED: $\quad$ Emergency department

EMS: $\quad$ Emergency medical services

ER: $\quad$ Emergency room

HUS: Helsinki University Hospital

PICU: $\quad$ Paediatric Intensive Care Unit

PPE: $\quad$ Personal protective equipment

SARS-CoV- Severe acute respiratory syndrome

2: $\quad$ coronavirus 2 .

\section{Data Availability}

Full laboratory findings are available from the authors upon request.

\section{Ethical Approval}

This case report does not have any intervention and did not influence the care of the patient. Thus, no ethics approval was needed. The permission to evaluate inhospital and out-of-hospital general data for patients with COVID-19 suspicion was granted by the institutional study protocol.

\section{Consent}

The patient's mother, who is also the legal representative of the patient, has given her written consent to publish the case, including results from laboratory tests and pictures, given that the patient cannot be recognized.

\section{Conflicts of Interest}

The authors declare no conflicts of interest.

\section{Authors' Contributions}

H. S., P. R., and J. R. H. were responsible for the care of the patient and wrote the case report. S. P. performed the bronchoscopy and critically reviewed the manuscript. H. H.R. commented on the prehospital care of the patient and critically reviewed the manuscript.

\section{Acknowledgments}

The writing was funded by institutional support only. The publishing of the article was funded by the Paediatric Research Center of New Children's Hospital, Helsinki, Finland (in January 2021, to the author H.S.).

\section{References}

[1] X. Lu, L. Zhang, H. Du et al., "SARS-CoV-2 infection in children," New England Journal of Medicine, vol. 382, no. 17, pp. 1663-1665, 2020.

[2] Y. Dong, X. Mo, Y. Hu et al., "Epidemiology of COVID-19 among children in China," Pediatrics, vol. 145, no. 6, Article ID e20200702, 2020.

[3] R. Castagnoli, M. Votto, A. Licari et al., "Severe acute respiratory syndrome coronavirus 2 (SARS-CoV-2) infection in children and adolescents: a systematic review," JAMA Pediatrics, 2020.

[4] N. Parri, M. Lenge, and D. Buonsenso, "Children with covid19 in pediatric emergency departments in Italy," New England Journal of Medicine, vol. 383, no. 2, p. 187, 2020.

[5] S. Garazzino, C. Montagnani, D. Donà et al., "Multicentre Italian study of SARS-CoV-2 infection in children and adolescents, preliminary data as at 10 April 2020," Eurosurveillance, vol. 25, no. 18, 2020.

[6] Q. Wu, Y. Xing, L. Shi et al., "Coinfection and other clinical characteristics of COVID-19 in children," Pediatrics, vol. 146, no. 1, Article ID e20200961, 2020.

[7] M. Lazzerini, E. Barbi, A. Apicella, F. Marchetti, F. Cardinale, and G. Trobia, "Delayed access or provision of care in Italy resulting from fear of COVID-19," The Lancet Child \& Adolescent Health, 2020.

[8] P. Green, "Risks to children and young people during COVID-19 pandemic," BMJ, Article ID m1669, 2020.

[9] E. Crawley, M. Loades, G. Feder, S. Logan, S. Redwood, and J. Macleod, "Wider collateral damage to children in the UK because of the social distancing measures designed to reduce the impact of COVID-19 in adults," BMJ Paediatrics Open, vol. 4, no. 1, Article ID e000701, 2020.

[10] M. Oncel, G. S. Sunam, and S. Ceran, "Tracheobronchial aspiration of foreign bodies and rigid bronchoscopy in children," Pediatrics International, vol. 54, no. 4, pp. 532-535, 2012.

[11] T. Adjeso, M. C. Damah, J. P. Murphy, and T. T. K. Anyomih, "Foreign body aspiration in northern Ghana: a review of pediatric patients," International Journal of Otolaryngology, vol. 2017, Article ID 1478795, 4 pages, 2017.

[12] T. A. Sultan and A. B. van As, "Review of tracheobronchial foreign body aspiration in the South African paediatric age group," Journal of Thoracic Disease, vol. 8, no. 12, pp. 3787-3796, 2016. 